\title{
AVALIAÇÃO DA BIOMASSA DE RAÍZES FINAS EM ÁREA DE EMPRÉSTIMO SUBMETIDA A DIFERENTES COMPOSIÇÕES DE ESPÉCIES ${ }^{1}$
}

\author{
Ricardo Valcarcel ${ }^{2}$, Flávio Dias Wanderley Valente ${ }^{2}$, Maira Jardineiro Morokawa², Felipe Vieira Cunha \\ Neto $^{2}$ e Carlos Rodrigues Pereira ${ }^{2}$
}

\begin{abstract}
RESUMO - O comportamento do sistema radicular dentro de um processo de construção de solo em área degradada varia entre indivíduos, espécies e comunidades. Para analisar esse comportamento, foi conduzido um estudo na região denominada Costa Verde, Município de Itaguaí, Estado do Rio de Janeiro, onde, utilizandose subsolo de área de empréstimo com características edafoclimáticas similares, submetida a três diferentes composições de espécies, cujas funções podem influenciar a construção dos solos e dos ecossistemas, encontrouse igualdade de produção total de raízes finas em $30 \mathrm{~cm}$ de profundidade, embora a vegetação com maior diversidade funcional e de espécies tenha contribuído mais para a construção dos ecossistemas.
\end{abstract}

Palavras-chave: Raízes finas, reabilitação e área degradada.

\section{EVALUATION OF FINE ROOT BIOMASS ON A RECLAMATION AREA UNDER DIFFERENT SPECIES COMPOSITIONS}

\begin{abstract}
The behavior of root system in the process of soil construction in degraded areas varies among individuals, species and communities. To evaluate this behavior, a study was conducted in a region named Costa Verde, municipal district of Itaguaí, State of Rio de Janeiro, using subsoil from a reclamation area with similar soil-climatic characteristics subjected to three different species compositions that can influence the ecosystem and soil construction. There was no difference for total fine root production at $30 \mathrm{~cm}$ depth, although the vegetation with the greatest functional and species diversity most contributed to ecosystem construction.
\end{abstract}

Keywords: Fine roots, rehabilitation and degraded area.

\section{INTRODUÇÃO}

A supressão da vegetação e a retirada abrupta do substrato promove a degradação dos ecossistemas com graves conseqüências para a sociedade, constituindo passivos ambientais a serem resgatados pelas gerações subseqüentes. Para que esses passivos sejam minimizados, eles precisam ser mitigados durante a exploração dos recursos, onde se faz necessário desenvolver estratégias específicas e eficazes de reabilitação de áreas degradadas.

As técnicas de reabilitação de áreas degradadas são variadas e estão em constante aperfeiçoamento. Intervêm no curto, médio e longo prazo, com o propósito de dotar os ambientes de propriedades emergentes para que eles alcancem a auto-sustentabilidade dos processos de construção dos ecossistemas. Uma das

\footnotetext{
${ }^{1}$ Recebido em 26.05.2006 e aceito para publicação em 24.05.2007.

${ }^{2}$ Laboratório de Manejo de Bacias Hidrográficas da Universidade Federal Rural do Rio de Janeiro. E-mail: <ricval@ufrrj.br>; <fdwalente@hotmail.com>; <maira-morokawa@hotmail.com>;<fvcneto@pop.com.br>; <carlosrodriguespereira@yahoo.com.br>
} 
técnicas utilizadas é o plantio de espécies arbóreas com a finalidade de reabilitação de ecossistemas degradados, conhecida como medida biológica (VALCARCELED'ALTÉRIO, 1998; VALCARCELeSILVA, 2000).

O crescimento e desenvolvimento dos sistemas radiculares dependem das interações entre fatores bióticos e abióticos dos ecossistemas (FELDMAN, 1988; FREITAS et al., 2005). Comportamentos de sistemas radiculares de espécies invasoras em terrenos cultiváveis são diferentes daqueles em terrenos impactados. Cada espécie arbórea pode desenvolver sistema radicular distinto, estando este condicionado à oferta de condições ambientais (PRITCHETT, 1979).

Os estudos de desenvolvimento de solos a partir dos sistemas radiculares, buscando a compreensão das funções e das influências dos componentes do meio biótico nas propriedades físico-químicas do solo, são recentes (FELDMAN, 1988). A quantidade e a extensão das raízes que se desenvolvem em cada condição ambiental está diretamente relacionada com as características físicas e químicas do solo, com os fatores genéticos das plantas, com o balanço da relação entre a parte aérea e a parte radicular, com o manejo do solo e com as práticas culturais adotadas (COSTA et al., 1999; JOHN et al., 2002; HERTEL et al., 2003).

As raízes influenciam as características do solo através da excreção de açúcares, ácidos orgânicos e compostos minerais de fósforo e potássio, contribuindo para a dissolução de substâncias minerais e para o desenvolvimento de microrganismos na rizosfera (CINTRA et al., 1999). Sua decomposição e renovação compõem o ciclo do carbono influenciando, desse modo, a manutenção da fertilidade (LEHMANN e ZECH, 1998; SCHROTH et al., 1999). Segundo estudos (LEHMANN e ZECH, 1998; SCHROTH et al., 1999), enquanto de 10 a $20 \%$ da serrapilheira é transformada em matéria orgânica do solo, estes valores podem variar entre 20 e $50 \%$ quando se considera a renovação das raízes.

O conhecimento das inter-relações que ocorrem entre os compartimentos de um ecossistema em construção é imprescindível para o aperfeiçoamento das técnicas de reabilitação de áreas degradadas (VALCARCEL e D'ALTÉRIO, 1998), entre eles a relação entre diversidade de espécies e seus efeitos dentro do substrato. Este estudo objetivou avaliar a quantidade de raízes finas em $30 \mathrm{~cm}$ de subsolo sob efeito de duas medidas biológicas (MB) de reabilitação durante 8 anos e de uma regeneração espontânea (testemunha) com 24 anos.

\section{MATERIAL E MÉTODOS}

A área de estudo situa-se na região denominada Costa Verde, distrito da Ilha da Madeira, Município de Itaguaí, Estado do Rio de Janeiro (23 55' 07' ' S e $43^{\circ} 50^{\prime} 35^{\prime \prime}$ W), aos fundos da Baía de Sepetiba, região de domínio ecológico da Mata Atlântica. É uma área de empréstimo (10,81 ha) de onde foram retirados 1.400 .000 $\mathrm{m}^{3}$ de substrato para a construção do porto de Itaguaí (profundidade média de $13 \mathrm{~m}$ ).

A área, que não teve intervenção conservacionista para reintegração da paisagem local desde 1980 até 1993, gerou ecossistemas frágeis que não subsistem às chuvas torrenciais, apresentando intensos processos erosivos.

No período compreendido entre 11 de outubro e 12 de novembro de 1994, as áreas foram reflorestadas com nove espécies com funções ecológicas distintas (Quadro 1): MB- $\alpha$, com uma espécie pioneira exótica (100\% dos indivíduos); e MB- $\beta$, com oito espécies, sendo uma pioneira exótica (15\%), seis pioneiras nativas ( $70 \%)$ e uma secundária nativa (15\% dos indivíduos). A testemunha foi constituída de plantas oriundas de regeneração espontânea.

As áreas apresentavam características edafo-climáticas similares, mesmas distâncias das fontes de propágulos, plantios eqüianos (10 anos) e espaçamentos, cuja densidade se encontrava próxima a 1.660 indivíduos/ha.

As raízes foram coletadas em 10 pontos aleatórios entre árvores no centro de cada parcela permanente $\left(350 \mathrm{~m}^{2}\right)$, no dia 12 de agosto de 2004. Utilizou-se anel volumétrico $\left(140 \mathrm{~cm}^{3}\right)$ nas profundidades $0-10,10-20$ e $20-30 \mathrm{~cm}$. As amostras foram acondicionadas em sacos plásticos e guardadas em geladeira até a triagem, conforme recomendado por Hertel et al. (2003).

As raízes finas $(\varnothing \leq 2 \mathrm{~mm})$ foram separadas do substrato por lavagem e secadas ao ar com o auxílio de tamizadores, conforme descrito na literatura (SCHROTH e KOLBE, 1994). O material separado foi classificado em vivo ou morto, utilizando critérios visuais (cor, grau de coesão entre a periderme e o córtex) e mecânicos (elasticidade, estabilidade), de acordo com John et al (2002). As raízes foram divididas em muito finas $(\varnothing<1 \mathrm{~mm})$ e finas $(1 \leq \varnothing \leq 2 \mathrm{~mm})$. 
Quadro 1 - Composição de espécies, com as funções ecológicas de pioneiras exóticas (PE), pioneiras nativas (PN) e secundárias nativas (SN), plantadas, como medidas biológicas alfa (MB- $\alpha$ ) e beta (MB- $\beta$ ), na área de empréstimo da Ilha da Madeira Itaguaí, RJ

Table 1 - Species composition, with ecological functions of exotic pioneer (PE), native pioneer (PN), secondary native $(S N)$, planted as biological measures alpha $(M B-\alpha)$ and beta $(M B-\beta)$ in the reclamation area, Ilha da Madeira Itaguaí, $R J$

\begin{tabular}{|c|c|c|c|c|}
\hline Espécies & MB- $\alpha$ & MB- $\beta$ & Composição (\%) & Função Ecológica \\
\hline Acacia auriculiformis Sandw & $\mathrm{X}$ & & 100 & $\mathrm{PE}$ \\
\hline Cecropia pachystachya Trec. & & $\mathrm{X}$ & 8 & PN \\
\hline Clitoria fairchildiana Howard & & $\mathrm{X}$ & 17 & $\mathrm{PN}$ \\
\hline Inga laurina (Sw.) Wild. & & $\mathrm{X}$ & 15 & $\mathrm{SN}$ \\
\hline Leucena leucocephala Spreng & & $\mathrm{X}$ & 15 & PE \\
\hline Mimosa bimucronata (DC.) Kuntze & & $\mathrm{X}$ & 14 & PN \\
\hline Mimosa caesalpiniaefolia Benth & & $\mathrm{X}$ & 20 & PN \\
\hline Piptadenia gonoacantha Mart. & & $\mathrm{X}$ & 5 & PN \\
\hline Schinus terebinthifolius Raddi & & $\mathrm{X}$ & 6 & PN \\
\hline Total de Espécies & 1,0 & 8,0 & & \\
\hline
\end{tabular}

Após triagem e classificação, o material foi secado em estufa a $70{ }^{\circ} \mathrm{C}$ até atingir peso constante. A massa seca das raízes finas $\left(\mathrm{kg} \cdot \mathrm{ha}^{-1}\right)$ foi obtida por pesagem em balança analítica.

Os parâmetros taxa de decomposição da serrapilheira (k), tempo médio de renovação da serrapilheira $\left(\frac{1}{k}\right)$, tempo necessário para a decomposição de $50 \%$ da serrapilheira $\left(\mathrm{t}_{0,5}\right)$ e tempo necessário para a decomposição de $95 \%$ da serrapilheira $\left(\mathrm{t}_{0,05}\right)$ (anos) foram obtidos segundo a metodologia descrita na literatura (ANDERSON e SWIFT, 1983; PAGANO, 1989).

Foram aplicados os testes de Kruskal-Wallis $(\mathrm{p}=0,05)($ não-paramétrico) e o teste de Tukey, para efetuar as comparações entre matéria seca de serrapilheira e de raízes finas vivas e mortas entre tratamentos. Utilizou-se o software estatístico SAEG (UFV, 1997).

\section{RESULTADOS E DISCUSSÃO}

A massa seca total de raízes ( $\mathrm{kg} \mathrm{MS} \cdot \mathrm{ha}^{-1} .30 \mathrm{~cm}^{-1}$ ) nas medidas biológicas não diferiu daquela observada na testemunha, embora se tenha observado tendência de maior produção e raízes nas medidas biológicas (Quadro 2). Os valores 924, 955 e 849 kg MS.ha-1.30 $\mathrm{cm}^{-1}$ para MB- $\alpha, \mathrm{MB}-\beta$ e testemunha, respectivamente, não apresentaram diferenças significativas entre tratamentos a 5\%, pelo teste de Tukey. Esses resultados não excluem os efeitos conservacionistas dos reflorestamentos como medida de reabilitação de áreas degradadas, pois o período de nove anos na realidade envolve dois ou três anos de efetiva influência da vegetação, uma vez que o período de estabelecimento foi de aproximadamente 5 anos.
A massa seca de raízes finas mortas (RFM) e vivas (RFV) apresentaram diferenças significativas na MB$\alpha$, onde a massa seca de RFV foi cinco vezes inferior à da MB- $\beta$ e testemunha. A massa seca de RFM foi significativamente maior em MB- $\alpha$.

A distribuição eqüitativa entre raízes vivas e mortas revela uma estratégia de adaptação das espécies às condições adversas, pois sua senescência, seguida da imobilização biológica, retém nutriente (GONÇALVES e MELLO, 2000). O desbalanço entre RFV/RFM apresentado na MB- $\alpha$ pode estar revelando menor adaptação das espécies ao ambiente com exíguos atributos ambientais no subsolo, fato esse corroborado pela menor entrada de espécies colonizadoras espontâneas sob o dossel, o que representa menor oferta de propriedades emergentes ao meio (VALCARCEL e SILVA, 2000; NEVES e VALCARCEL, 2000).

Quadro 2 - Matéria seca (MS) (kg MS.ha-1 $\left.30 \mathrm{~cm}^{-1}\right)$ de raízes finas vivas ( $\mathrm{RFV}$ ) e de raízes finas mortas (RFM) amostrada nos $30 \mathrm{~cm}$ de profundidade do solo (substrato), nas medidas biológicas e na testemunha

Table 2 -Dry matter (MS) ( $\left.\mathrm{kg} \mathrm{MS.} \mathrm{ha}^{-1} .30 \mathrm{~cm}^{-1}\right)$ from live fine roots $(R F V)$ and from dead fine roots ( $R F M)$ sampled on soil at $30 \mathrm{~cm}$ depth (substrate), in the biological measures and in the control

\begin{tabular}{lccc}
\hline Estado das raízes & \multicolumn{3}{c}{ Tratamentos } \\
\cline { 2 - 4 } & Medida & Medida & Testemunha \\
& Biológica $\alpha$ & Biológica $\beta$ & \\
\hline Raízes Finas Vivas & $70 \pm 17^{\mathrm{b}}$ & $352 \pm 86^{\mathrm{a}}$ & $317 \pm 60^{\mathrm{a}}$ \\
Raízes Finas Mortas & $854 \pm 140^{\mathrm{b}}$ & $603 \pm 102^{\text {a }}$ & $532 \pm 82^{\mathrm{a}}$ \\
\hline
\end{tabular}

OBS: Valores seguidos da mesma letra na mesma linha não diferem significativamente entre si, pelo teste de Tukey $(\mathrm{p}=0,05 ; \mathrm{N}=30)$.

R. Árvore, Viçosa-MG, v.31, n.5, p.923-930, 2007 
A distribuição porcentual de RFV e RFM (Figura 1) indica que a competição intra-específica prejudicou o desenvolvimento radicular, visto que a MB- $\alpha$ é um plantio homogêneo de Acacia auriculiformes.

A distribuição da massa seca radicular nas profundidades amostradas foi diferente nos tratamentos (Figura 2). Na MB- $\alpha$, a massa seca foi significativamente superior na camada superficial $(0-10 \mathrm{~cm})$ em relação às demais. Na MB- $\beta$ houve diferença entre a camada superficial e a de $20-30 \mathrm{~cm}$. A testemunha não apresentou diferenças significativas na produção de massa seca entre as diferentes profundidades.

Entre tratamentos, não houve diferença significativa quando analisados em cada profundidade (Figura 2). As áreas reflorestadas tiveram menos massa seca que a testemunha, nas profundidades de $10-20 \mathrm{~cm}$ e de $20-30 \mathrm{~cm}$. O declínio na quantidade de raízes produzidas com o aumento da profundidade apresentou formato diferente nos três tratamentos. Os efeitos das medidas biológicos, como agentes construtores de ecossistemas, foram evidenciados nos primeiros $10 \mathrm{~cm}$ de profundidade, no perfil do substrato.

A função das espécies que compõem esses ecossistemas é variável no tempo, pois depende do alcance da maturidade das espécies. Durante os 10 anos ocorreram efeitos distintos sobre o substrato, influindo na distribuição radicular. A exposição diferenciada à irradiância interfere no desenvolvimento ecofisiológico das espécies e do sub-bosque, gerando produção diferenciada de serrapilheira e componentes inorgânicos (Quadro 3), afetando o funcionamento dos ecossistemas (TIENNE et al., 2003; VALENTE et al., 2005) e o desenvolvimento das raízes.

O tipo de raiz das plantas pode ter interferido na distribuição radicular no perfil do solo (substrato),1 uma vez que algumas espécies de plantas C4 (capim em geral) possuem raiz fasciculada, e isso implica uma produção maior de massa radicular; contrário ao caso das plantas com raiz pivotante (árvores), que produzem menor quantidade de raiz lateralmente. Outro ponto a ser considerado é a razão parte aérea/parte radicular, pois a produção e distribuição radicular constituem função também da demanda evapotranspirométrica. A maior concentração na camada superficial, nos tratamentos em relação à testemunha, pode ser explicada pelo microambiente favorável causado pela serrapilheira, com maior retenção de água, maior arejamento e, portanto, maior disponibilidade de oxigênio e disponibilidade de nutrientes oriundos de sua decomposição.

Os efeitos oriundos da produção total anual de serrapilheira, oito vezes maior nas MBs que na testemunha, não foram suficientes para provocar diferenças no comportamento do sistema radicular nos $30 \mathrm{~cm}$ de profundidade, em 10 anos. Os 24 anos em que a parcela-testemunha ficou submetida à colonização de poucas espécies herbáceas que se adaptaram ao meio, com alta amplitude térmica diária, indicaram que a escolha das espécies é fundamental para a reabilitação de áreas degradadas, principalmente se os processos erosivos não forem intensos.

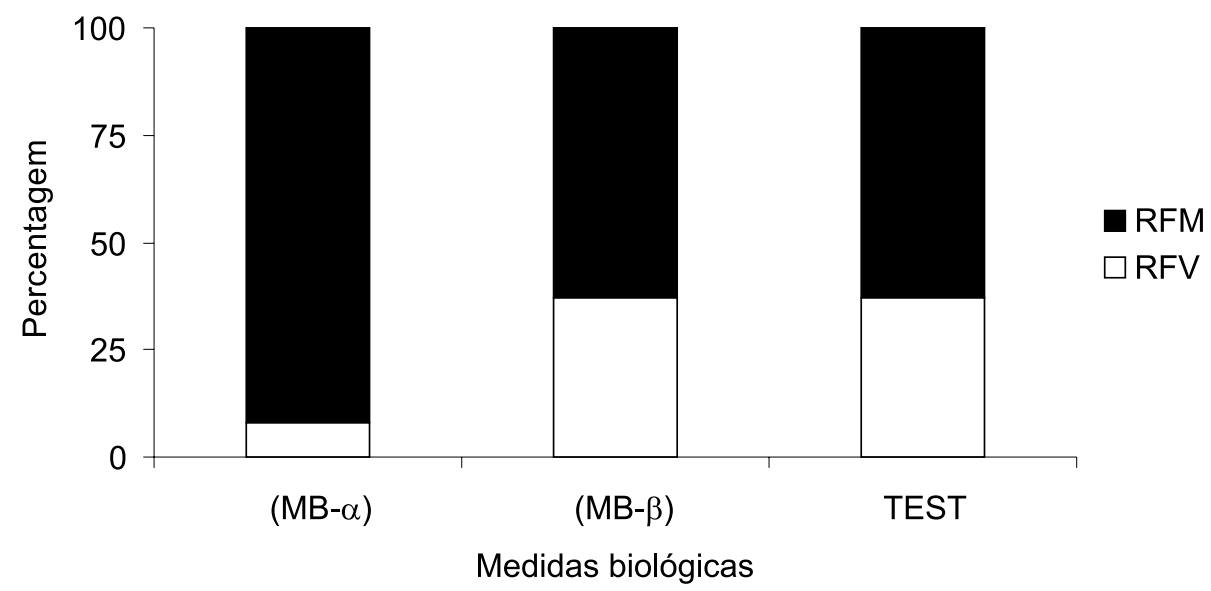

Figura 1 - Porcentagem de raízes finas vivas (RFV) e de raízes finas mortas (RFM) verificadas nos tratamentos medida biológica $\alpha$ (MB- $\alpha$ ), medida biológica $\beta$ (MB- $\beta$ ) e testemunha (TEST), respectivamente.

Figure 1 - Percentage of live fine roots $(R F V)$ and dead fine roots (RFM) found in the treatments biological measures $\alpha$ $(M B-\alpha)$, biological measures $\beta(M B-\beta)$ and control (TEST), respectively.

R. Árvore, Viçosa-MG, v.31, n.5, p.923-930, 2007 


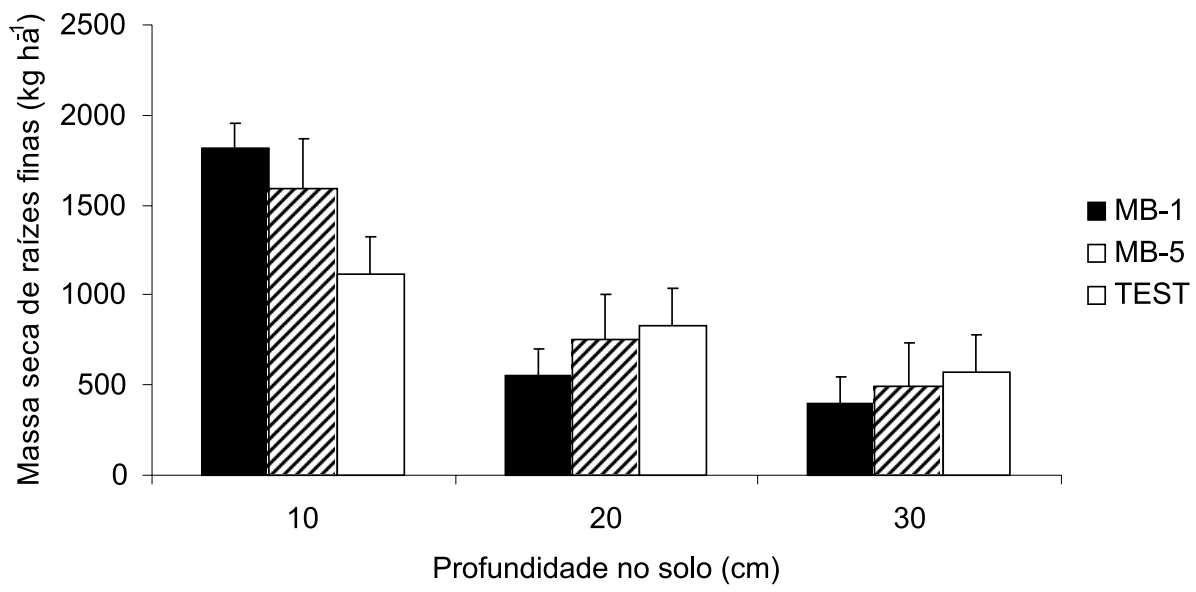

Figura 2 - Massa seca de raízes com diâmetros menores ou iguais a $2 \mathrm{~mm}$ (finas), verificada no perfil do solo (substrato) em diferentes profundidades, nos tratamentos medida biológica $\alpha$ (MB- $\alpha$ ), medida biológica $\beta$ (MB- $\beta$ ) e testemunha (TEST), respectivamente.

Figure 2 -Dry matter of roots with diameters below or equal to two millimeters (fine) found in the soil profile (substrate) at different depths, for the treatments biological measure $\alpha(M B-\alpha)$, biological measure $\beta(M B-\beta)$ and control (TEST), respectively.

Quadro 3 - Matéria seca, em quilogramas por hectare (kg $\left.\mathrm{ha}^{-1}\right)$, das diferentes partes das plantas que compuseram a serrapilheira acumulada no período de 2002-2003, nas medidas biológicas e testemunha

Table 3 - Dry matter, in kilograms per hectare $\left(\mathrm{kg} \mathrm{ha}^{-1}\right)$, from different parts of the plant that composed the litter accumulated in the period 2002-2003, in the biological measures and control

\begin{tabular}{lccc}
\hline Componentes & \multicolumn{3}{c}{ Tratamentos } \\
\cline { 2 - 4 } da serrapilheira & $\begin{array}{c}\text { Medida } \\
\text { Biológica } \alpha\end{array}$ & $\begin{array}{c}\text { Medida } \\
\text { Biológica } \beta\end{array}$ & Testemunha \\
\hline Galhos & $1531^{\mathrm{a}}$ & $1285^{\mathrm{b}}$ & $96^{\mathrm{c}}$ \\
Folhas & $6621^{\mathrm{b}}$ & $6447^{\mathrm{b}}$ & $751^{\mathrm{c}}$ \\
Flores & $713^{\mathrm{b}}$ & $1899^{\mathrm{a}}$ & $130^{\mathrm{b}}$ \\
Sementes & $584^{\mathrm{a}}$ & $307^{\mathrm{ab}}$ & $74^{\mathrm{b}}$ \\
Frutos & $1058^{\mathrm{b}}$ & $721^{\mathrm{c}}$ & $104^{\mathrm{c}}$ \\
Resíduo & $391^{\mathrm{b}}$ & $785^{\mathrm{a}}$ & $150^{\mathrm{b}}$ \\
\hline Total & $10898^{\mathrm{c}}$ & $11444^{\mathrm{bc}}$ & $1305^{\mathrm{d}}$ \\
\hline
\end{tabular}

OBS.: Valores seguidos da mesma letra na mesma linha não diferem significativamente entre si, pelo teste de Tukey $(p=0,05)$.

O sombreamento provocado pela árvores influenciou negativamente a ocorrência da vegetação de porte herbáceo das áreas onde foram introduzidas as $\mathrm{MBs}$, reduzindo as raízes nos 20 e $30 \mathrm{~cm}$ de profundidade nas medidas biológicas MB- $\alpha$ e MB$\beta$ em relação à testemunha. $\mathrm{Na} M B-\beta$, a diversidade de espécies contribuiu para melhorar a distribuição de raízes ao longo do perfil (Figura 2).
Esses resultados coincidem com os encontrados por John et al. (2002) em estudo de biomassa e decomposição de raízes em povoamento de Pinus kesiya. Os referidos autores encontraram maior biomassa de raízes finas na camada superficial do solo em todas as parcelas dos povoamentos com diferentes idades. Resultados semelhantes foram encontrados em povoamentos de Eucalyptus sp. (WITSCHORECK et al., 2003). Hertel et al. (2003) observaram em solo mineral $(0-10 \mathrm{~cm})$, de florestas tropicais, maior densidade de raízes em ambientes cuja manta orgânica era mais espessa. A biomassa de raízes observada na manta orgânica foi superior àquela encontrada no solo mineral nos ambientes evoluídos, enquanto na área em regeneração mais recente a maior parte das raízes foi encontrada no solo mineral, com $88 \%$ da biomassa ocorrendo nessa camada.

Esses resultados evidenciam o papel das condições microclimáticas no desenvolvimento do sistema radicular, pois as plantas, ao estabelecerem relações com a serrapilheira e suas camadas subseqüentes, concentram o desenvolvimento das raízes finas, principais responsáveis pela absorção de água e nutrientes nessas regiões, aproveitando ao máximo os nutrientes liberados pela ciclagem, além de contarem com maior disponibilidade de oxigênio nessa camada.

R. Árvore, Viçosa-MG, v.31, n.5, p.923-930, 2007 
A fração de raízes muito finas $(\varnothing<1 \mathrm{~mm})$ predominou em todas as profundidades dos tratamentos (Figura $3)$. Estas responderam por mais de $60 \%$ do conteúdo total de raízes em todos os tratamentos e profundidades. Tais resultados estão de acordo com aqueles registrados na literatura e, provavelmente, se devem à exígua capacidade de os ecossistemas mineralizarem os componentes orgânicos, uma vez que a mesofauna é incipiente e a matéria orgânica, de baixa degradabilidade (HERTEL et al., 2003). As espécies mais ajustadas evolutivamente a esses ecossistemas apresentam composição de biomassa radicular que permite melhor sobrevivência.

A importância das raízes finas na absorção de nutrientes e a alta concentração de raízes mortas indicam que a maioria das raízes vivas deve ter sido recentemente formada. Outro fator importante nesse aspecto é que a maior porcentagem de raízes muito finas na profundidade de $30 \mathrm{~cm}$ foi verificada na testemunha, indicando que só se estabeleceram na área espécies capazes de explorar locais mais profundos do perfil do substrato, sendo, nesse caso, a MB- $\alpha$ a menos eficiente.

Uma floresta nativa tropical com mais de 200 anos apresentou duas vezes mais raízes finas vivas que raízes finas mortas (HERTEL et al., 2003). No processo de construção de ecossistema auto-sustentável a partir da área de empréstimo, os resultados foram contrários a estes em todos os tratamentos (Figura 1). Na MB$\alpha$, o conteúdo de raízes finas vivas foi cerca de 10 vezes inferior ao conteúdo de raízes finas mortas. Esse dado pode ser justificado pela época de coleta das amostras, correspondendo ao fim do período chuvoso quando, geralmente, ocorrem mais morte e conseqüente diminuição das raízes vivas (LARCHER, 2000).

A MB- $\beta$ apresentou conteúdo de raízes nas camadas de 10-20 e 20-30 cm superior ao da MB- $\alpha$ (Figura 2). Esse resultado pode estar relacionado com a maior diversidade de espécies e atributos ambientais desses ecossistemas (NEVES e VALCARCEL, 2000), indicando uma ocupação diferenciada do perfil pelas diferentes espécies, o que ocorre em menor escala na MB- $\alpha$ (plantio homogêneo). Tais resultados, porém, não apresentaram diferenças estatísticas significativas.

O tratamento composto de maior diversidade de espécies (MB- $\beta$ ) se mostrou mais eficiente apresentando substrato com maior fixação de carbono (Quadro 4) e melhores desempenhos quanto à taxa de decomposição (k), tempo médio de renovação da serrapilheira $\left(\frac{1}{k}\right)$, tempo necessários para a decomposição de $50 \%$ da serrapilheira $\left(\mathrm{t}_{0.5}\right)$ e tempo necessário para a decomposição de $95 \%$ da serrapilheira $\left(\mathrm{t}_{0,05}\right)$ (Quadro 5).
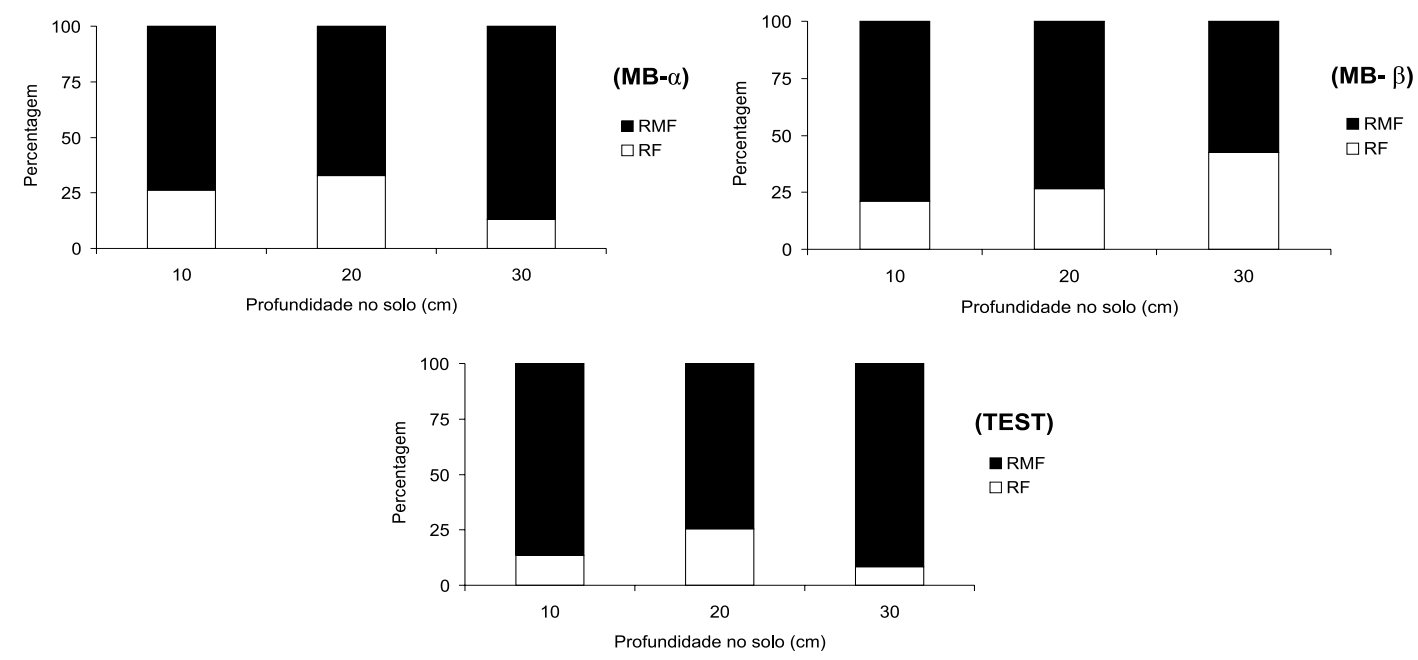

Figura 3 - Porcentagem de raízes finas (RF-diâmetro maior ou igual a $1 \mathrm{~mm}$ e menor ou igual a $2 \mathrm{~mm}$ ) e de raízes muito finas (RFM-diâmetro menor que $1 \mathrm{~mm}$ ) distribuídas no perfil do solo (substrato), nos tratamentos medida biológica $\alpha(\mathrm{MB}-\alpha)$, medida biológica $\beta$ (MB- $\beta$ ) e testemunha (TEST), respectivamente.

Figure 3 - Percentage of fine roots (RF-diameter greater or equal to one millimeter and smaller or equal to two millimeters) and very fine roots (RFM-diameters below one millimeter) distributed in the soil profile (substrate) for the treatments biological measures $\alpha(M B-\alpha)$, biological measure $\beta(M B-\beta)$ and control (TEST) respectively.

R. Árvore, Viçosa-MG, v.31, n.5, p.923-930, 2007 
Quadro 4-Resultados analíticos de amostras de solo (substratos) onde foram implantadas as diferentes medidas biológicas na área de empréstimo, após cinco anos de plantio e na testemunha

Table 4-Analytical results from soil samples (substrates) in which the different biological measures were implanted, in the reclamation area, five years postplanting, and control

\begin{tabular}{lccc}
\hline Análise Química & \multicolumn{3}{c}{ Resultados } \\
\cline { 2 - 4 } & $\begin{array}{c}\text { Medida } \\
\text { Biológica } \alpha\end{array}$ & $\begin{array}{c}\text { Medida } \\
\text { Biológica } \beta\end{array}$ & Testemunha \\
\hline pH em água 1:25 & 5,5 & 5,4 & 5,1 \\
$\mathrm{P}(\mathrm{ppm})$ & 6,0 & 6,0 & 2,0 \\
$\mathrm{~K}(\mathrm{ppm})$ & $>156$ & $>156$ & 87 \\
$\mathrm{Al}^{3+}(\mathrm{meq} / 100 \mathrm{~mL})$ & 1,0 & 1,0 & 1,0 \\
$\mathrm{Ca}^{2+}(\mathrm{meq} / 100 \mathrm{~mL})$ & 0,5 & 1,0 & 0,5 \\
$\mathrm{Mg}^{2+}(\mathrm{meq} / 100 \mathrm{~mL})$ & 1,0 & 1,0 & 0,3 \\
$\mathrm{H}+\mathrm{Al}(\mathrm{meq} / 100 \mathrm{~mL})$ & 0,8 & 0,8 & 1,5 \\
$\mathrm{Na}(\mathrm{meq} / 100 \mathrm{~mL})$ & 0,04 & 0,04 & 0,02 \\
$\mathrm{C}(\%)$ & 0,2 & 1,4 & 0,4 \\
\hline
\end{tabular}

Quadro 5-Parâmetros da serrapilheira, taxa de decomposição (k), tempo médio de renovação da serrapilheira (1/k), tempo necessário para a decomposição de $50 \%$ da serrapilheira $\left(\mathrm{t}_{0.5}\right)$ e tempo necessário para a decomposição de $95 \%$ da serrapilheira $\left(\mathrm{t}_{0,05}\right)(\mathrm{anos})$

Table 5 - Litter parameters rate of decomposition ( $k$ ), average time of litter renovation $(1 / k)$, time necessary to decompose $50 \%$ of litter $\left(t_{0.5}\right)$ and time necessary to decompose $95 \%$ of litter $\left(t_{0,05}\right)$ (years)

\begin{tabular}{cccc}
\hline Parâmetros & \multicolumn{3}{c}{ Tratamentos } \\
\cline { 2 - 4 } da Serrapilheira & $\begin{array}{c}\text { Medida } \\
\text { Biológica } \alpha\end{array}$ & $\begin{array}{c}\text { Medida } \\
\text { Biológica } \beta\end{array}$ & Testemunha \\
\hline$k$ & 0,82 & 2,03 & 1,43 \\
$\frac{1}{k}$ & 1,22 & 0,49 & 0,70 \\
$t 0,5$ & 0,85 & 0,34 & 0,48 \\
$t 0,05$ & 3,67 & 1,48 & 2,10 \\
\hline
\end{tabular}

\section{CONCLUSÃO}

O povoamento com maior diversidade de espécies e funções (MB- $\beta$ ) se mostrou mais equilibrado na produção de raízes finas, sendo mais eficiente nos processos auto-sustentáveis de construção de solos e de reabilitação de áreas degradadas.

\section{AGRADECIMENTOS}

Ao CNPq, Pedreira Sepetiba Ltda. e Laboratório de Manejo de Bacias Hidrográficas da Universidade Federal Rural do Rio de Janeiro.

\section{REFERÊNCIAS}

ANDERSON, J.M.; SWIFT, M.J. Decomposition in tropical forests. In: SUTTON, S.L.; WHITMORE, T.C.; CHADWICK, A.C. (Eds.) Tropical rain forest: ecology and management. London: Blackwell Scientific, 1983. p.287-309.

CINTRA, F.L.D.; LIBARDI, P.L.; JORGE, L.A.C. Distribuição do sistema radicular do citros em solo de tabuleiro costeiro. In: WORKSHOP SOBRE SISTEMA RADICULAR: METODOLOGIAS E ESTUDOS DE CASOS, 1999, Aracajú Anais...Aracajú, 1999. p.179-189.

COSTA, A.; ROSOLEM, C.A.; TORRES, H. Distribuição de raízes de leguminosas em função de alterações nas características químicas e físicas em solos do Paraná . In: WORKSHOP SOBRE SISTEMA RADICULAR: METODOLOGIAS E ESTUDOS DE CASOS, 1999, Aracajú Anais...Aracajú, 1999. p.191-202.

FELDMAN, L.J. The habits of roots. BioScience, v.38, n.9, p.612-618, 1988.

FREITAS, T.A.S. et al. Desempenho radicular de mudas de eucalipto produzidas em diferentes recipientes e substratos. Revista Árvore, v.29, n.6, p.853-861, 2005.

GONÇALVES, J.L.M.; MELLO, S.L.M. O sistema radicular das árvores. In: Nutrição e fertilização de florestas. Piracicaba: IPEF, 2000. p.221-267.

HERTEL, D.; LEUSCHNER, C.; HÖLSCHER, D. Size and structure of fine root systems in old-growth and secondary tropical montane forests (Costa Rica). Biotropica, v.35, n.2, p.143-153, 2003.

JOHN, B.; PANDEY, H.N.; TRIPATHI, R.S.

Decomposition of fine roots of Pinus Kesiya and turnover of organic matter, $\mathrm{N}$ and $\mathrm{P}$ of coarse and fine pine roots and herbaceous roots and rhizomes in subtropical pine forest stands of different ages. Biology and Fertility of Soils, v.35, p.238-246, 2002.

R. Árvore, Viçosa-MG, v.31, n.5, p.923-930, 2007 
LEHMANN, J.; ZECH, W. Fine root turnover of irrigated hedgerow intercropping in Northern Kenya. Plant and Soil, v.198, p.19-31, 1998.

LARCHER, W. Ecofisiologia vegetal. São Paulo: Rima Artes e Textos, 2000. 531p.

NEVES, L.G.; VALCARCEL, R. Regeneração natural em áreas de empréstimo em vias de reabilitação. In: JORNADA DE INICIAÇÃO CIENTÍFICA DA UFRRJ, 10., 2000,_Seropédica. Resumos..., Seropédica: 2000. p.157-158.

PAGANO, S.N. Produção de serrapilheira em mata mesófila semi-decídua no município de Rio Claro, SP. Revista Brasileira de Biologia, v.49, p.633-639, 1989.

\section{PRITCHeTt, W.L. Properties and}

management of forest soils. New York: John Wiley \& Sons, 1979. 500 p.

SCHROTH, G. et al. Root research methods for humid tropical agro-forestry systems - A management perspective. In: WORKSHOP SOBRE SISTEMA RADICULAR: METODOLOGIAS E ESTUDOS DE CASOS, 1999, Aracajú. Anais... Aracajú: 1999. p.255-268.

SCHROTH, G.; KOLBE, D. A method of processing soil core samples for root studies by subsampling. Biology and Fertility of Soils, v.18, p.60-62, 1994.
TIENNE, L. et al. Monitoramento de medidas biológicas em reabilitação de áreas de empréstimo: análise da projeção e cobertura de copa na Ilha da Madeira, Itaguaí-RJ. In: JORNADA DE INICIAÇÃO CIENTÍFICA DA UFRRJ, 13., 2003, Seropédica, 2003. Anais... Seropédica: 2003. p.233-238.

UNIVERSIDADE FEDERAL DE VIÇOSA SAEG: sistema de análises estatísticas e genéticas. Viçosa, MG: 1997. 150p. (Manual do usuário- versão 7.1).

VALENTE, F.D.V. et al. Produção e decomposição de serrapilheira em medidas biológicas de reabilitação de áreas de empréstimo na Mata Atlântica. Revista Universidade Rural, (aceito para publicação).

VALCARCEL, R.; D’ALTÉRIO, C.F. Medidas físico-biológicas de recuperação de áreas degradadas: avaliação das modificações edáficas e fitossociológicas. Floresta e Ambiente, v.5, n.1, 68-88, 1998.

VALCARCEL, R.; SILVA, Z. A eficiência conservacionista de medidas de recuperação de áreas degradadas: proposta metodológica. Floresta, v.27, n.1, p.101-114, 2000.

WITSCHORECK, R.; SCHUMACHER, M.V.; CALDEIRA, M.V.W. Estimating of biomass and length of fine roots in Eucalyptus urophylla S.T. Blake in the county of Santa Maria, RS. Revista Árvore, v.27, n.2, p.177-183, 2003. 